\title{
Numerical simulation of thermal fracture in functionally graded materials using element-free Galerkin method
}

\author{
SAHIL GARG*(D) and MOHIT PANT \\ Department of Mechanical Engineering, National Institute of Technology, Hamirpur 177005, India \\ e-mail: sahil.garg1017@gmail.com
}

MS received 11 February 2016; revised 9 May 2016; accepted 6 October 2016

\begin{abstract}
In the present work, element-free Galerkin method (EFGM) has been extended and implemented to simulate thermal fracture in functionally graded materials. The thermo-elastic fracture problem is decoupled into two separate parts. Initially, the temperature distribution over the domain is obtained by solving the heat transfer problem. The temperature field so obtained is then employed as input for the mechanical problem to determine the displacement and stress fields. The crack surfaces are modelled as non-insulated boundaries; hence the temperature field remains undisturbed by the presence of crack. A modified conservative $M$-integral technique has been used in order to extract the stress intensity factors for the simulated problems. The present analysis shows that the results obtained by EFGM are in good agreement with those available in the literature.
\end{abstract}

Keywords. EFGM; functionally graded material; intrinsic enrichment; M-integral; crack; SIF.

\section{Introduction}

Most of the structures and machine components are subjected to adverse thermo-mechanical working environments. Quite often these components are required to fulfill different requirements at different locations within the same component. This fuelled the development of a new class of materials called functionally graded materials (FGMs). The FGM concept originated in 1984 in Japan [1] during a space-plane project, in the form of a thermal barrier material capable of withstanding a surface temperature of $2000 \mathrm{~K}$ and a temperature gradient of $1000 \mathrm{~K}$ across a cross section $<10 \mathrm{~mm}$. FGMs were initially designed as thermal barrier materials for aerospace structural components. They are now being developed for general use as structural components in extremely high temperature environments. The goal of producing such engineered material systems is to utilize the strength of each material constituent at specific locations. The significant proportions of an FGM contain the pure form of each component, and the need for compromise is eliminated by properly utilizing the properties of each component. For example, the toughness of a metal can be mated with the refractoriness of a ceramic, without any compromise in the toughness of the metal side or the refractoriness of the ceramic side. FGMs and FGM coating are used in wide variety of applications [2-5]. FGMs offer great promise in applications where the operating conditions are quite severe, e.g., wear-resistant linings for handling large heavy abrasive ore particles, rocket heat

*For correspondence shields, heat exchanger tubes, thermoelectric generators, heat-engine components, plasma facings for fusion reactors, piezoelectric devices, graded refractive index materials, thermionic converters, dental and other implants, fireretardant doors, solid oxide fuel cell and electrically insulating metal-ceramic joints. FGMs are also ideal for minimizing the thermo-mechanical mismatch in metal-ceramic bonds.

\section{Fracture in FGMs}

The microstructure of FGMs is generally heterogeneous in nature and the dominant type of failure is crack initiation and growth from inclusions. FGMs are characterized by gradual spatial variation in composition, microstructure and material properties of each component. A continuous gradation leads to the reduction of residual stresses developed at the interface of bi-material systems developed for the same purpose. However, in practice FGMs are often composed of a number of layers in which the volume fraction of second phase increases with addition of each layer. As a result the interface stresses get redistributed across multiple layers. The extent to which constituent material properties and microstructure of FGMs can be altered remains an area of keen interest. This has lead to a lot of analytical and computational research in the area of fracture behaviour of FGMs. Analytical work on FGMs goes back to 1960s with modelling of soil as a non-homogeneous material [6]. Many researchers solved crack problems for non-homogeneous 
materials subjected to mechanical loading [7-9]. The asymptotic crack tip stress field in FGMs is found to possess the square root singularity as that of homogeneous material [9]. The thermal stress intensity factors (SIFs) for non-homogeneous solids were computed [10] by assuming an exponential variation of thermal properties. Erdogan [11], while analysing the fracture mechanics of FGMs, established that FGM coatings can reduce residual and thermal stresses. Gu and Asaro [12] analysed a semi-infinite crack in a strip of FGM. They obtained the SIFs (SIFs) for commonly used fracture specimens. An equivalent domain integral technique was presented [13] for calculating the SIF for FGMs using finite-element method (FEM), where the material property variation is achieved by assigning different homogeneous elastic properties to different elements. Chen et al [14] coined a new form of $J$-integral, which possessed the path-independent property in case of FGMs. Both experimental and FEM analyses were carried out [15] for a crack lying normal to elastic gradient. Kim and Paulino [16] evaluated the mixed-mode fracture parameters in FGMs using FEM. The analysis of FGM coatings was performed in many works by computational methods, usually FEM, or analytical tools [10, 17-20]. Some other approaches to simplify the mechanics of FGMs were developed like using equivalent eigenstrain and distributed dislocation technique to symbolize the cracks by Afsar and Song [21]. Since the computational methods saved plenty of engineering effort and provided more accuracy, some meshless approaches were developed to counteract the shortcomings of conventional FEM solutions. Element-free Galerkin method (EFGM) was employed [22, 23] to solve the problems involving cracks in isotropic FGM and to conduct thermomechanical analysis of FGMs, respectively. Later on extended finite-element method (XFEM) was used for analysis of FGMs [24], which was extended to 3D analysis [25] and investigation of orthotropic FGMs [26].

Though a considerable development in the field of fracture analysis of FGMs has been achieved by various approaches, still to the best of author's knowledge, no significant work has been performed for simulating thermal fracture of FGM using EFGM. Motivated by the wide applicability and advantages of FGMs over conventional materials, the present work is an effort to extend the application of EFGM in simulating the fracture of FGMs under mechanical-thermal loads.

\section{Overview of EFGM}

In EFGM, the field variable $u$ is approximated by moving least-square approximation function $u^{h}(\mathbf{x})$, which is given by [27]

$$
u^{h}(\mathbf{x})=\sum_{j=1}^{m} p_{j}(\mathbf{x}) a_{j}(\mathbf{x})=\mathbf{p}^{T}(\mathbf{x}) \mathbf{a}(\mathbf{x})
$$

where $\mathbf{p}(\mathbf{x})$ is a vector of basis functions, $\mathbf{a}(\mathbf{x})$ are unknown coefficients and $m$ is the number of terms in the basis. The unknown coefficients $\mathbf{a}(\mathbf{x})$ are obtained by minimizing a weighted least-square sum of the difference between local approximation, $u^{h}(\mathbf{x})$ and field function nodal parameters $u_{I}$. The weighted least-square sum $L(\mathbf{x})$ can be written in the following quadratic form:

$$
L(\mathbf{x})=\sum_{I=1}^{n} w\left(\mathbf{x}-\mathbf{x}_{I}\right)\left[\mathbf{p}^{T}(\mathbf{x}) \mathbf{a}(\mathbf{x})-u_{I}\right]^{2}
$$

where $u_{I}$ is the nodal parameter (primary variable, displacement) associated with node $I$ at $\mathbf{x}=\mathbf{x}_{I}$. However, $u_{I}$ are not the nodal values of $u^{h}\left(\mathbf{x}=\mathbf{x}_{I}\right)$ since $u^{h}(\mathbf{x})$ is the approximated value of nodal parameter (using EFG shape functions) and not an interpolated one as in the case of FEM. The difference between $u_{I}$ and $u^{h}\left(\mathbf{x}=\mathbf{x}_{I}\right)$ is shown in figure $1 ; w\left(\mathbf{x}-\mathbf{x}_{I}\right)$ is the weight function having compact support associated with node $I$, and $n$ is the number of nodes with domain of influence containing the point $\mathbf{x}$, i.e. $w\left(\mathbf{x}-\mathbf{x}_{I}\right) \neq 0$.

By setting $\partial L / \partial \mathbf{a}=0$, following set of linear equation is obtained:

$$
\mathbf{A}(\mathbf{x}) \mathbf{a}(\mathbf{x})=\mathbf{B}(\mathbf{x}) \mathbf{u}
$$

where

$$
\mathbf{A}(\mathbf{x})=\sum_{I=1}^{n} w\left(\mathbf{x}-\mathbf{x}_{I}\right) \mathbf{p}\left(\mathbf{x}_{I}\right) \mathbf{p}^{T}\left(\mathbf{x}_{I}\right)
$$

$\mathbf{B}(\mathbf{x})=\left\{w\left(\mathbf{x}-\mathbf{x}_{1}\right) \mathbf{p}\left(\mathbf{x}_{1}\right), w\left(\mathbf{x}-\mathbf{x}_{2}\right) \mathbf{p}\left(\mathbf{x}_{2}\right), \ldots, w\left(\mathbf{x}-\mathbf{x}_{n}\right) \mathbf{p}\left(\mathbf{x}_{n}\right)\right\}$.

Using Eqs. (3) and (1), the approximation function is obtained as

$$
u^{h}(\mathbf{x})=\sum_{I=1}^{n} \Phi_{I}(\mathbf{x}) u_{I}
$$

where $\Phi_{I}(\mathbf{x})=\sum_{j=}^{m} p_{j}(\mathbf{x})\left(\mathbf{A}^{-1}(\mathbf{x}) \mathbf{B}(\mathbf{x})\right)_{j I}=\mathbf{p}^{T} \mathbf{A}^{-1} \mathbf{B}_{I}$.

The linear consistency requirements for the shape function $\Phi_{I}(\mathbf{x})$ are given as follows [28]:

$$
\sum_{I=1}^{n} \Phi_{I}(\mathbf{x})=1, \quad \sum_{I=1}^{n} \Phi_{I}(\mathbf{x}) x_{I}=x, \quad \sum_{I=1}^{n} \Phi_{I}(\mathbf{x}) y_{I}=y .
$$

The derivatives of MLS shape function are computed as follows:

$$
\begin{aligned}
\Phi_{I, \mathbf{x}}(\mathbf{x}) & =\left(\mathbf{p}^{T} \mathbf{A}^{-1} \mathbf{B}_{I}\right)_{, \mathbf{x}} \\
& =\mathbf{p}_{, \mathbf{x}}^{T} \mathbf{A}^{-1} \mathbf{B}_{I}+\mathbf{p}^{T}\left(\mathbf{A}^{-1}\right)_{, \mathbf{x}} \mathbf{B}_{I}+\mathbf{p}^{T} \mathbf{A}^{-1} \mathbf{B}_{I, \mathbf{x}}
\end{aligned}
$$

where $\mathbf{B}_{I, \mathbf{x}}(\mathbf{x})=\frac{d w}{d \mathbf{x}}\left(\mathbf{x}-\mathbf{x}_{I}\right) \mathbf{p}\left(\mathbf{x}_{I}\right)$ and $\mathbf{A}_{, \mathbf{x}}^{-1}$ is computed by 


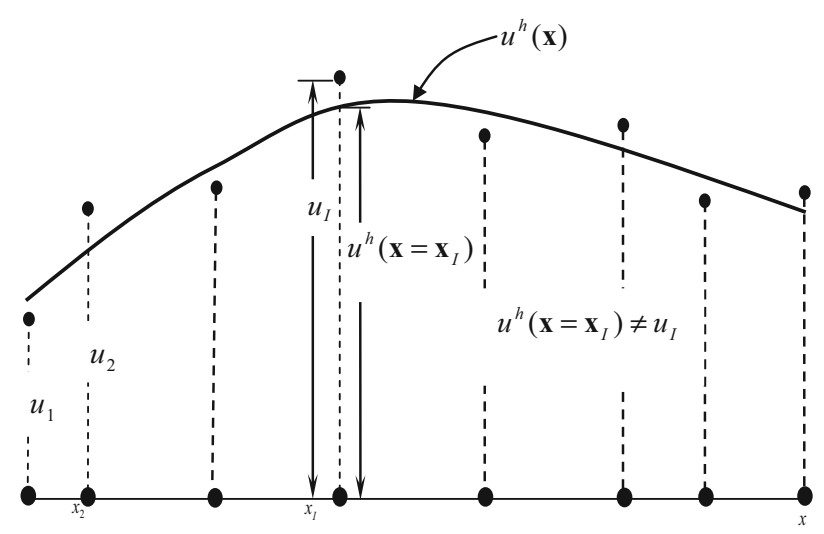

Figure 1. Difference between $u_{I}$ and $u^{h}(\mathbf{x})$.

$$
\mathbf{A}_{, \mathbf{x}}^{-1}=-\mathbf{A}^{-1} \mathbf{A}_{, \mathbf{x}} \mathbf{A}^{-1}
$$

where

$$
\mathbf{A}_{, \mathbf{x}}=\sum_{I=1}^{n} \frac{d w}{d \mathbf{x}}\left(\mathbf{x}-\mathbf{x}_{I}\right) \mathbf{p}\left(\mathbf{x}_{I}\right) \mathbf{p}^{T}\left(\mathbf{x}_{I}\right) .
$$

\subsection{Weight functions}

The choice of weight function $w\left(\mathbf{x}-\mathbf{x}_{I}\right)$ affects the resulting approximation $u^{h}\left(\mathbf{x}_{I}\right)$ in EFGM. Therefore, the selection of appropriate weight function becomes quite important. The weight function is non-zero only over a small neighbourhood of a node $\mathbf{x}_{I}$, called the support or domain of influence of node $I$. The smoothness and continuity of the shape function $\Phi_{I}(\mathbf{x})$ depends on the smoothness and continuity of the weight function $w\left(\mathbf{x}-\mathbf{x}_{I}\right)$. If a weight function is $C^{1}$ continuous then the shape function will also have $C^{1}$ continuity. In the present work a cubic spline weight function [29] has been used, which is given as follows:

$$
w\left(\mathbf{x}-\mathbf{x}_{I}\right)=w(\bar{r})=\left\{\begin{array}{ll}
\frac{2}{3}-4 \bar{r}^{2}+4 \bar{r}^{3} & \bar{r} \leq \frac{1}{2} \\
\frac{4}{3}-4 \bar{r}+4 \bar{r}^{2}-\frac{4}{3} \bar{r}^{3} & \frac{1}{2}<\bar{r} \leq 1 \\
0 & \bar{r}>1
\end{array}\right\}
$$

where $\bar{r}=\frac{\left\|\mathbf{x}-\mathbf{x}_{I}\right\|}{d_{m I}},\left\|\mathbf{x}-\mathbf{x}_{I}\right\|$ is the distance from a sampling point $\mathbf{x}$ to a node $\mathbf{x}_{I}, d_{m I}$ is the influence domain of node $I$, $\bar{r}=\frac{\left\|\mathbf{x}-\mathbf{x}_{I}\right\|}{d_{m I}}, d_{m I}=d_{\max } c_{I}, d_{\max }$ is a scaling parameter that defines size of the domain of influence, $c_{I}$ at node $I$ is the distances to the nearest neighbours and $d_{m I}$ is chosen such that the matrix is non-singular at every point in the domain.

\section{EFGM formulation for FGMs}

Consider a two-dimensional domain with small displacements, bounded by $\Gamma$ (figure 2). It is assumed that the material properties such as modulus of elasticity $E$, Poisson's ratio $v$, thermal conductivity $k$ and coefficient of thermal expansion $\beta$ vary as follows:

$$
\begin{gathered}
E=E\left(x_{1}, x_{2}\right)=E(x) \\
v=v\left(x_{1}, x_{2}\right)=v(x) \\
k=k\left(x_{1}, x_{2}\right)=k(x) \\
\beta=\beta\left(x_{1}, x_{2}\right)=\beta(x) .
\end{gathered}
$$

The governing equilibrium equations [27] are given as

$$
\nabla . \boldsymbol{\sigma}+\mathbf{b}=0 \text { in } \Omega
$$

with the following essential and natural boundary conditions:

$$
\begin{gathered}
\mathbf{u}=\overline{\mathbf{u}} \text { on } \Gamma_{u} \quad(\text { essential }) \\
\boldsymbol{\sigma} \cdot \overline{\mathbf{n}}=\overline{\mathbf{t}} \text { on } \Gamma_{t} \quad \text { (natural) }
\end{gathered}
$$

where $\boldsymbol{\sigma}$ is the stress tensor, which is defined as $\boldsymbol{\sigma}=\mathbf{D}(\mathbf{x})\left[\boldsymbol{\varepsilon}-\boldsymbol{\varepsilon}_{T}\right], \mathbf{D}(\mathbf{x})$ is the material property matrix, $\boldsymbol{\varepsilon}$ is the strain vector, $\boldsymbol{\varepsilon}_{T}=\beta \Delta T$ is the thermal strain vector, $\mathbf{b}$ is the body force vector, $\mathbf{u}$ is the displacement vector, $\overline{\mathbf{t}}$ is the traction force and $\overline{\mathbf{n}}$ is the unit normal.

By enforcing essential boundary conditions using a Lagrange multiplier approach, and applying variational principle, the following discrete equations are obtained from Eqs. (4) and (13):

$$
\left[\begin{array}{cc}
\mathbf{K} & \mathbf{G} \\
\mathbf{G}^{T} & 0
\end{array}\right]\left\{\begin{array}{l}
\mathbf{u} \\
\boldsymbol{\lambda}
\end{array}\right\}=\left\{\begin{array}{l}
\mathbf{f} \\
\mathbf{q}
\end{array}\right\}
$$

where

$$
\begin{gathered}
K_{I J}=\int_{\Omega} \mathbf{B}_{I}^{T} \mathbf{D} \mathbf{B}_{I} d \Omega \\
f_{I}=\int_{\Gamma_{t}} \overline{\mathbf{t}} \Phi_{I} d \Gamma_{t}, \quad q_{K}=-\int_{\Gamma_{u}} \mathbf{N}_{K} \bar{u} d \Gamma_{u},
\end{gathered}
$$

$\mathbf{N}_{K}$ is a one-dimensional Lagrange interpolant

$$
\mathbf{B}_{I}=\left[\begin{array}{cc}
\Phi_{I, x} & 0 \\
0 & \Phi_{I, y} \\
\Phi_{I, y} & \Phi_{I, x}
\end{array}\right], \quad \mathbf{N}_{K}=\left[\begin{array}{cc}
N_{K} & 0 \\
0 & N_{K}
\end{array}\right]
$$




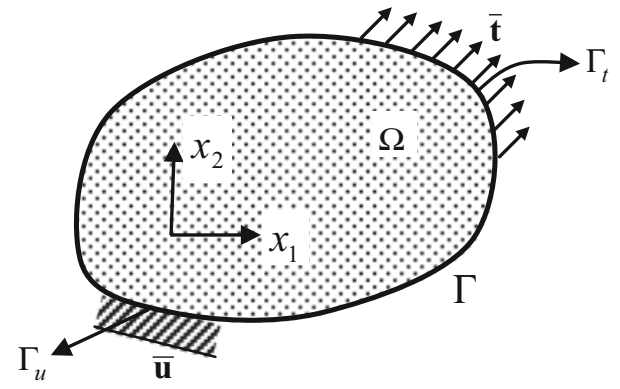

Figure 2. Domain along with essential and natural boundary conditions.

$$
\mathbf{D}(x)=\frac{E(x)}{1-v(x)^{2}}\left[\begin{array}{ccc}
1 & v(x) & 0 \\
v(x) & 1 & 0 \\
0 & 0 & (1-v(x)) / 2
\end{array}\right]
$$

(for plane stress)

$$
\begin{aligned}
& =\frac{E(x)}{[1+v(x)][(1-2 v(x))]} \\
& {\left[\begin{array}{ccc}
1-v(x) & v(x) & 0 \\
v(x) & 1-v(x) & 0 \\
0 & 0 & (1-2 v(x)) / 2
\end{array}\right]}
\end{aligned}
$$

(for plane strain).

In FGMs, the elasticity matrix $\mathbf{D}(x)$ is spatially dependent because of graded material properties. The effect of material gradation on stiffness matrix $\mathbf{K}$ can be incorporated directly by calculating the material properties at Gauss points lying over the domain.

\section{Thermal interaction integral for FGMs}

Similar to monolithic materials, the $J$-integral approach can also be used to compute the stress intensities for FGMs; however, it must account for material gradients. Generally, during formulation of interaction integral, terms such as derivatives of the elastic constants are discarded. For a monolithic material, the derivatives of elastic constants are zero, and have no contribution in calculation of stress intensities. However, for the case of a continuously graded material, these terms are non-zero and must be retained in order to maintain the path independence of the $J$-integral. Several formulations of interaction integral for non-homogeneous materials have been developed [22, 30, 31]. In the present work, thermal interaction integral will be used to account for both mechanical-thermal loading. For a homogenous cracked body, the path independent $J$-integral [32] is given as

$$
J=\int_{\Gamma}\left(\tilde{W} \delta_{1 j}-\sigma_{i j} \frac{\partial u_{i}}{\partial x_{1}}\right) n_{j} d \Gamma
$$

where $\tilde{W}$ is the strain energy density and $n_{j}$ is the $j$ th component of the outward unit vector normal to an arbitrary contour $\Gamma$ enclosing the crack tip (figure 3 ). For linear elastic materials subjected to thermal loads.

$$
\tilde{W}=\frac{\sigma_{i j} \varepsilon_{i j}^{m}}{2}=\frac{1}{2} \sigma_{i j}\left(\varepsilon_{i j}^{t}-\beta \Delta T \delta_{i j}\right)
$$

where $\varepsilon_{i j}^{m}$ denotes the mechanical part of strain, $\varepsilon_{i j}^{t}$ the total strain, $\beta=\beta(x)$ the coefficient of thermal expansion that varies with spatial coordinates, $\Delta T=T-T_{0}$ where $T_{0}$ is the reference temperature and $\delta_{i j}$ is the Kronecker delta.

In order to enhance its usefulness, the contour integral in Eq. (21) can be converted into an equivalent domain form using divergence theorem [33]:

$$
\begin{aligned}
J= & \int_{A}\left(\sigma_{i j} \frac{\partial u_{i}}{\partial x_{1}}-\tilde{W} \delta_{1 j}\right) \frac{\partial \bar{q}}{\partial x_{j}} d A \\
& +\int_{A} \frac{\partial}{\partial x_{j}}\left(\sigma_{i j} \frac{\partial u_{i}}{\partial x_{1}}-\tilde{W} \delta_{1 j}\right) \bar{q} d A
\end{aligned}
$$

where $A$ is the area inside the contour and $\bar{q}$ is a weight function chosen such that it has a value unity at the crack tip, zero along the boundary of the domain and it is arbitrary elsewhere.

For calculating the interaction integral, two equilibrium states of a cracked body are considered. State 1 corresponds to be the actual state with the given boundary conditions while state 2 is defined to be an auxiliary state. The superposition of two states leads to another equilibrium state $S$ for which the domain form of the $J$-integral is given as follows [34]:

$$
\begin{aligned}
J^{s}= & \int_{A}\left\{\left(\sigma_{i j}+\sigma_{i j}^{a u x}\right)\left(u_{i, 1}+u_{i, 1}^{a u x}\right)-\frac{1}{2}\left(\sigma_{i k}+\sigma_{i k}^{a u x}\right)\left(\varepsilon_{i k}^{m}+\varepsilon_{i k}^{a u x}\right) \delta_{i j}\right\} \bar{q}_{, j} d A \\
& +\int_{A}\left\{\left(\sigma_{i j}+\sigma_{i j}^{a u x}\right)\left(u_{i, 1}+u_{i, 1}^{a u x}\right)-\frac{1}{2}\left(\sigma_{i k}+\sigma_{i k}^{a u x}\right)\left(\varepsilon_{i k}^{m}+\varepsilon_{i k}^{a u x}\right) \delta_{i j}\right\}_{, j} \bar{q} d A
\end{aligned}
$$

Equation (24) can be decomposed into

$$
J^{s}=J^{1}+J^{a u x}+M
$$

where the interaction integral $M$ is given by

$$
\begin{aligned}
M= & \int_{A}\left\{\sigma_{i j} u_{i, 1}^{a u x}+\sigma_{i j}^{a u x} u_{i, 1}-\frac{1}{2}\left(\sigma_{i k} \varepsilon_{i k}^{a u x}+\sigma_{i k}^{a u x} \varepsilon_{i k}^{m}\right) \delta_{1 j}\right\} \bar{q}_{, j} d A \\
& +\int_{A}\left\{\sigma_{i j} u_{i, 1}^{a u x}+\sigma_{i j}^{a u x} u_{i, 1}-\frac{1}{2}\left(\sigma_{i k}^{a u x}+\sigma_{i k}^{a u x} \varepsilon_{i k}^{m}\right) \delta_{1 j}\right\}_{, j} \bar{q} d A
\end{aligned}
$$

Based on non-equilibrium formulation for FGMs, we have

$$
\sigma_{i j} \varepsilon_{i j}^{a u x}=C_{i j k l}(x) \varepsilon_{k l}^{m} \varepsilon_{i j}^{a u x}=\sigma_{k l}^{a u x} \varepsilon_{k l}^{m}=\sigma_{i j}^{a u x} \varepsilon_{i j}^{m} .
$$

Again, rewrite Eq. (26) as 


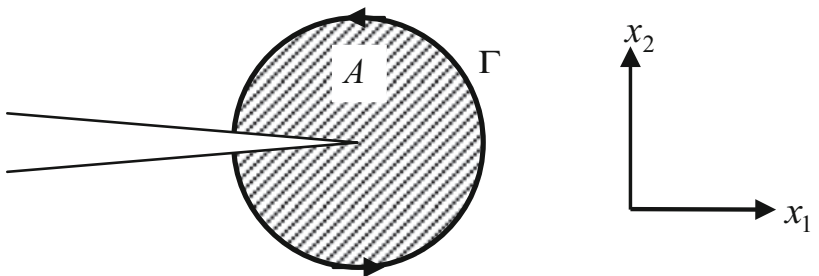

Figure 3. Path $\Gamma$ surrounding a crack with an enclosed area A.

$$
\begin{aligned}
M= & M_{1}+M_{2} \\
= & \int_{A}\left\{\sigma_{i j} u_{i, 1}^{a u x}+\sigma_{i j}^{a u x} u_{i, 1}-\sigma_{i k} \varepsilon_{i k}^{a u x} \delta_{1 j}\right\}_{, j} d A \\
& +\int_{A}\left\{\sigma_{i j} u_{i, 1}^{a u x}+\sigma_{i j}^{a u x} u_{i, 1}-\sigma_{i k} \varepsilon_{i k}^{a u x} \delta_{1 j}\right\}_{, j} \bar{q} d A .
\end{aligned}
$$

The last term of integral $M_{2}$ in Eq. (27) is expressed as

$$
\begin{aligned}
\left(\sigma_{i k} \varepsilon_{i k}^{a u x} \delta_{1 j}\right)_{, j} & =\left(\sigma_{i j} \varepsilon_{i j}^{a u x}\right)_{, 1}=\left(C_{i j k l} \varepsilon_{k l}^{m} \varepsilon_{i j}^{a u x}\right)_{, 1} \\
& =C_{i j k l, 1} \varepsilon_{k l}^{m} \varepsilon_{i j}^{a u x}+C_{i j k l} \varepsilon_{k l, 1}^{m} \varepsilon_{i j}^{a u x}+C_{i j k l} \varepsilon_{k l}^{m} \varepsilon_{i j, 1}^{a u x} \\
& =C_{i j k l, 1} \varepsilon_{k l}^{m} \varepsilon_{i j}^{a u x}+\sigma_{i j}^{a u x} \varepsilon_{i j, 1}^{m}+\sigma_{i j} \varepsilon_{i j, 1}^{a u x} .
\end{aligned}
$$

Substituting Eq. (28) into $M_{2}$ of Eq. (27) leads to

$$
\begin{aligned}
M_{2}= & \int_{A}\left(\sigma_{i j, j} u_{i, 1}^{a u x}+\sigma_{i j} u_{i, 1 j}^{a u x}+\sigma_{i j, j}^{a u x} u_{i, 1}+\sigma_{i j}^{a u x} u_{i, 1 j}\right) \bar{q} d A \\
& -\int_{A}\left(C_{i j k l, 1} \varepsilon_{k l}^{m} \varepsilon_{i j}^{a u x}+\sigma_{i j}^{a u x} \varepsilon_{i j, 1}^{m}+\sigma_{i j} \varepsilon_{i j, 1}^{a u x}\right) \bar{q} d A
\end{aligned}
$$

Using compatibility conditions (for actual and auxiliary state) and equilibrium condition (for actual state) i.e. $\sigma_{i j, j}=0$, Eq. (29) can be simplified as

$$
M_{2}=\int_{A}\left\{\sigma_{i j, j}^{a u x} u_{i, 1}-C_{i j k l, 1} \varepsilon_{k l}^{m} \varepsilon_{i j}^{a u x}+\sigma_{i j}^{a u x}\left(u_{i, 1 j}-\varepsilon_{i j, 1}^{m}\right)\right\} \bar{q} d A
$$

$=\int_{A}\left\{\sigma_{i j, j}^{a u x} u_{i, 1}-C_{i j k l, 1} \varepsilon_{k l}^{m} \varepsilon_{i j}^{a u x}+\sigma_{i j}^{a u x}\left(\beta_{, 1} \Delta T+\beta(\Delta T)_{, 1}\right) \delta_{i j}\right\} \bar{q} d A$.

Therefore, the resulting interaction integral $(M)$ becomes

$$
\begin{aligned}
M= & \int_{A}\left\{\sigma_{i j, j}^{a u x} u_{i, 1}-C_{i j k l, 1} \varepsilon_{k l}^{m} \varepsilon_{i j}^{a u x}+\sigma_{i j}^{a u x}\left(\beta_{, 1} \Delta T+\beta(\Delta T)_{, 1}\right) \delta_{i j}\right\} \bar{q} d A \\
& +\int_{A}\left\{\sigma_{i j} u_{i, 1}^{a u x}+\sigma_{i j}^{a u x} u_{i, 1}-\sigma_{i k} \varepsilon_{i k}^{a u x} \delta_{1 j}\right\} \bar{q}_{, j} d A
\end{aligned}
$$

where the term $\sigma_{i j} u_{i, 1}^{a u x}$ appears due to non-equilibrium of auxiliary stress fields.

The non-equilibrium formulation used in this work produces a very simple final form of $M$-integral. In terms of accuracy the results are as good as the incompatibility formulation and in comparison with constant constitutive tensor formulation this form can produce more efficient results [31].

\section{Evaluation of stress intensity factors}

For linear elastic solid under mixed-mode loading conditions, $J$-integral is also equal to the energy release rate and hence it can be written as follows:

$$
J=\frac{1}{E^{*}}\left(K_{I}^{2}+K_{I I}^{2}\right)
$$

where

$$
E^{*}=\left\{\begin{array}{cc}
\frac{E}{1-v^{2}} & \text { Plane strain } \\
E & \text { Plane stress }
\end{array}\right\} .
$$

Applying Eq. (33) to states 1 (actual), state 2 (auxiliary) and the superimposed state $S$ gives

$$
\begin{aligned}
J^{(1)} & =\frac{1}{E^{*}}\left(K_{I}^{(1)^{2}}+K_{I I}^{(1)^{2}}\right) \\
J^{(a u x)} & =\frac{1}{E^{*}}\left(K_{I}^{(a u x)^{2}}+K_{I I}^{(a u x)^{2}}\right)
\end{aligned}
$$

and

$$
\begin{aligned}
J^{(S)}= & \frac{1}{E^{*}}\left[\left(K_{I}^{(1)}+K_{I}^{(\text {aux })}\right)^{2}+\left(K_{I I}^{(1)}+K_{I I}^{(\text {aux })}\right)^{2}\right] \\
= & \frac{1}{E^{*}}\left[\left(K_{I}^{(1)^{2}}+K_{I I}^{(1)^{2}}\right)+\left(K_{I}^{(1)^{2}}+K_{I I}^{(a u x)^{2}}\right)\right. \\
& \left.+2\left(K_{I}^{(1)} K_{I}^{(\text {aux })}+K_{I I}^{(1)} K_{I I}^{(a u x)}\right)\right] \\
= & J^{(I)}+J^{(\text {aux })}+\frac{2}{E^{*}}\left(K_{I}^{(1)} K_{I}^{(\text {aux })}+K_{I I}^{(1)} K_{I I}^{(a u x)}\right) .
\end{aligned}
$$

Comparing Eq. (25) with Eq. (36) gives

$$
M=\frac{2}{E^{*}}\left[\left(K_{I}^{(1)} K_{I}^{(a u x)}+K_{I I}^{(1)} K_{I I}^{(a u x)}\right)\right] .
$$

The individual SIFs for the actual state can be obtained by judiciously choosing the auxiliary state (state 2). For example, if state 2 (auxiliary state) is chosen to be in modeI, i.e. mode-I near tip displacement, and stress field is chosen as the auxiliary state, then $K_{I}^{(2)}=1$ and $K_{I I}^{(2)}=0$. Hence, Eq. (37) can be reduced to

$$
M^{(1, I)}=\frac{2 K_{I}^{(1)}}{E_{t i p}^{*}}
$$


or

$$
K_{I}^{(1)}=\frac{M^{(1, I)} E_{t i p}^{*}}{2} .
$$

Similarly, if state 2 is chosen to be in mode-II, i.e. mode-II near tip displacement, and stress field is chosen as the auxiliary state, then $K_{I}^{(2)}=0$ and $K_{I I}^{(2)}=1$. Following similar considerations

$$
K_{I I}^{(1)}=\frac{M^{(1, I I)} E_{t i p}^{*}}{2} .
$$

Thus, numerical evaluation of interaction integral from Eq. (32) allows us to calculate the mixed-mode SIFs.

\section{Results and discussions}

Apart from a standard edge crack problem subjected to mode-I mechanical load, some additional problems have been solved to show the capability of EFGM in modelling and simulating thermal fracture in FGMs.

\subsection{Edge crack plate subjected to mode-i mechanical loading}

An edge crack plate of dimensions $H=8$ units, $W=1$ unit and a crack of length of $a$ is considered as shown in figure 4. The elastic modulus of the FGM plate is assumed to follow an exponential gradation as given by the function $E\left(x_{1}\right)=E_{1} \exp \left(\eta x_{1} / W\right), 0 \leq x_{1} \leq W$ with $E_{1}=E(0), E_{2}=$ $E(W)$ and $\eta=\ln \left(E_{2} / E_{1}\right)$. A uniform nodal distribution over the domain along with six-point Gauss quadrature is used in each cell. The results are obtained under plane

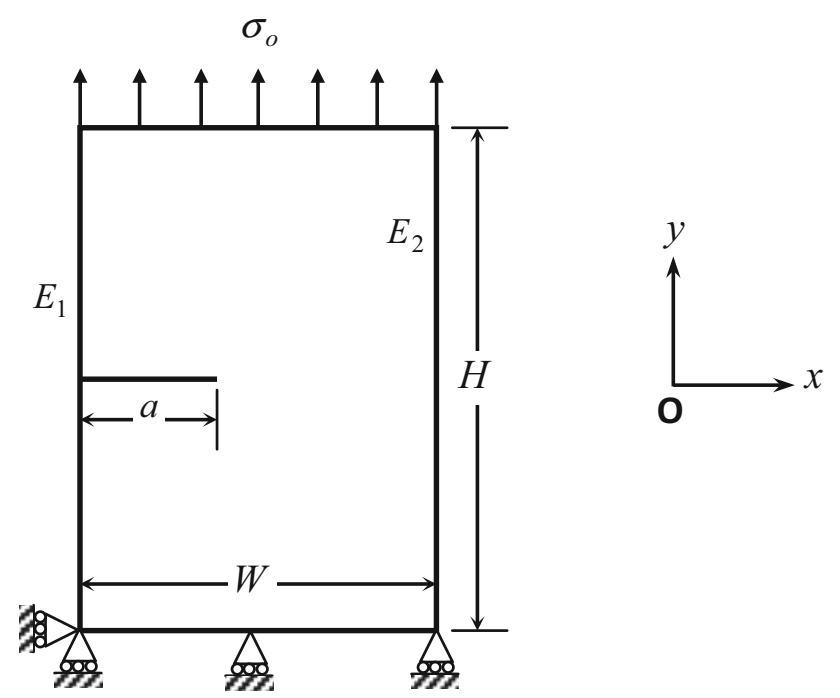

Figure 4. Problem geometry along with dimensions and boundary conditions. strain condition with $E_{1}=1$ unit, $v=0.3$ and $E_{2} / E_{1}=\exp (\eta)=0.1,0.2,5,10$ with a varying ratio of $a / W=0.2,0.3,0.4,0.5,0.6$. The values of mode-I stress intensity factor are normalized according to the relation $\tilde{F}_{I}=\frac{K_{I}}{\sigma_{o} \sqrt{\pi a}}$. Figure 5a shows the variation of normalized $\operatorname{SIF}\left(K_{I}\right)$ with varying $(a / W)$ ratio for $E_{2} / E_{1}=0.1$. Figure $5 \mathrm{~b}$ shows the variation of modulus of elasticity $(E)$. The results obtained by EFGM exhibit a good agreement with available solutions in Reference 1 [35] and in Reference 2 [36]. Similar results are obtained by varying the $E_{2} / E_{1}$ ratio as shown in figures $6 \mathrm{a}-8 \mathrm{a}$ along with the corresponding spatial variation of modulus of elasticity $(E)$ as shown in figures $6 b-8 b$.

\subsection{Edge crack plate subjected to thermal loading}

7.2a FGM with linear gradation of material properties: Next, an edge crack plate ( $W=1$ unit and $H=4$ units) subjected to thermal load is considered. The material properties of plate follow a linear variation from left edge to right edge. The geometrical dimensions of plate along with boundary conditions are shown in figure 9a. Temperature is prescribed on left and right edges, while the top and bottom edges are considered to be insulated. A uniform nodal distribution of 784 nodes along with six-point Gauss quadrature in each cell is used to simulate the problem. A plane strain condition is assumed over the problem domain. The values of mode-I SIFs, i.e. $K_{I}$, are calculated using domain-based interaction integral approach. The following numerical data are used in the present analysis: $E_{1}=$ $1 \times 10^{5}$ units, $E_{2}=0.5 \times 10^{5}$ units, $v_{1}=0.3, v_{2}=0.35$, $\beta_{1}=1.67 \times 10^{-5} /{ }^{\circ} \mathrm{C}$ units, $\beta_{2}=1 \times 10^{-5} /{ }^{\circ} \mathrm{C}$ units, $T_{1}=0{ }^{\circ} \mathrm{C}, T_{2}=1{ }^{\circ} \mathrm{C}$ and $T_{0}=0{ }^{\circ} \mathrm{C}$.

The subscripts " 1 " and " 2 " in this section represent values of material properties on left and right edges, respectively, while $T_{0}$ is the reference (ambient) temperature [34].

A plot of steady state temperature distribution is shown in figure 9b. This plot shows that the temperature distribution remains unaffected by the presence of the crack under constant heat flux, which is parallel to the crack surface [37]. The prescribed boundary conditions generate a pure mode-I loading as can be clearly seen from displaced nodal positions in figure 10a. The values of mode-I SIF, i.e. $K_{I}$, have been calculated for different $\left(\frac{a}{W}\right)$ ratios as shown in figure 10b. The results obtained by EFGM are found to be in good agreement with those obtained by FEM [34].

7.2b FGM with hyperbolic-tangent gradation of material properties: An edge cracked functionally graded plate with material properties variation defined by hyperbolic-tangent function is analysed in this section. Figure 11a shows the dimensions ( $W=2$ units and $H=4$ units) of FGM plate along with an edge crack of length $a$. The top and bottom edges are constrained in the $y$-direction. The problem 


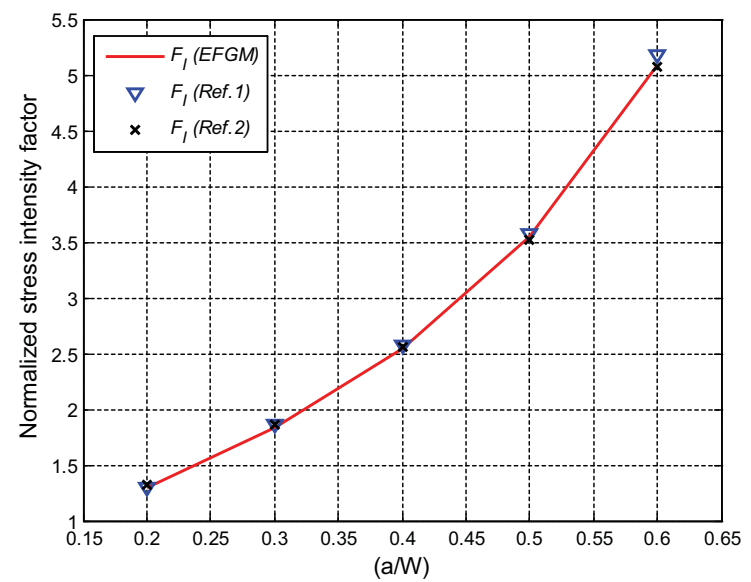

(a)

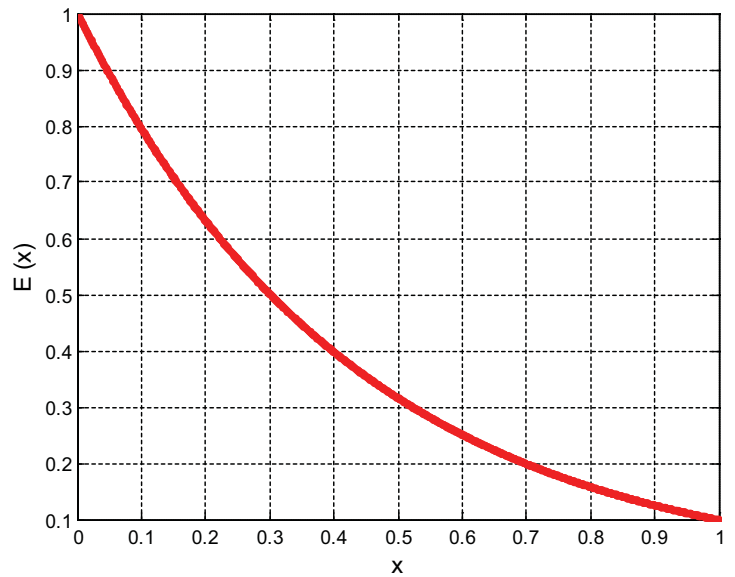

(b)

Figure 5. SIF variation and modulus of elasticity gradation for $E_{2} / E_{1}=0.1$.

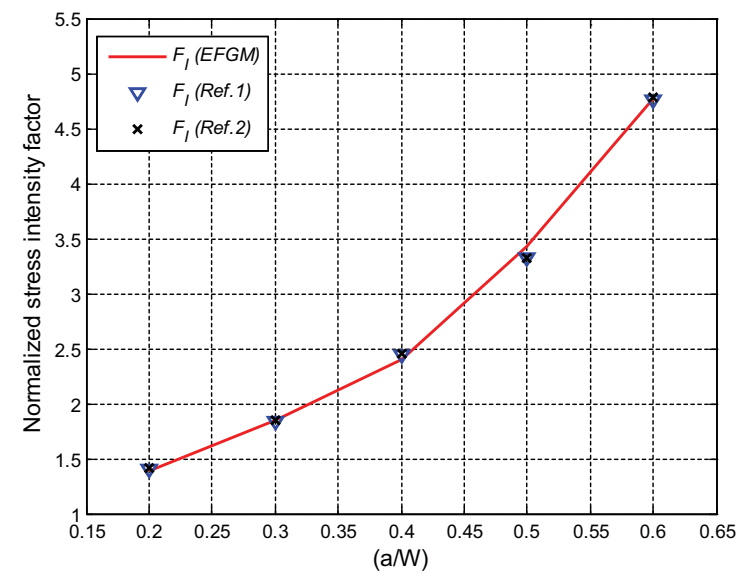

(a)

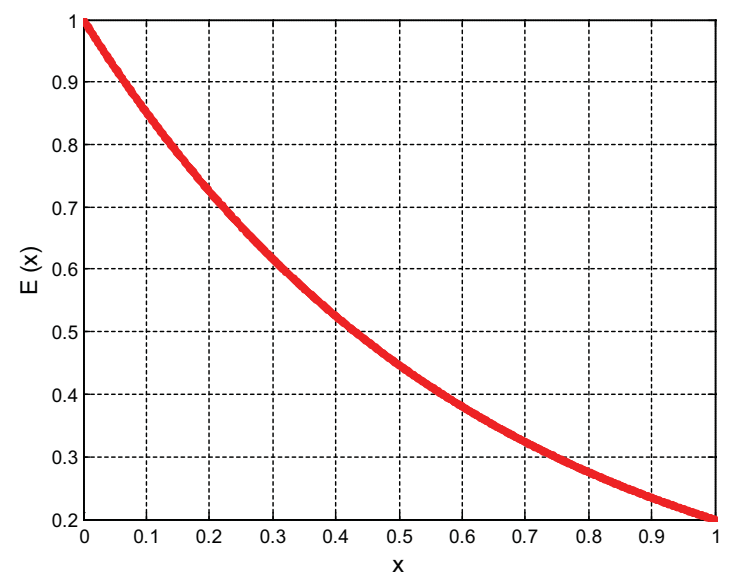

(b)

Figure 6. SIF variation and modulus of elasticity gradation for $E_{2} / E_{1}=0.2$.

domain is subjected to a steady state thermal loading having $T_{1}=-10^{\circ} \mathrm{C}$ and $T_{2}=0^{\circ} \mathrm{C}$. The top and bottom edges are considered to be insulated so that there is no flow of heat flux across them. A uniform arrangement of 1250 nodes along with six-point Gauss quadrature has been used to simulate the problem under plane strain conditions. The gradation of Young's modulus $(E)$, Poisson's ratio $(v)$ and thermal expansion coefficient $(\beta)$ are defined by hyperbolic-tangent function as follows:

$$
\begin{gathered}
E\left(X_{1}\right)=\frac{E^{-}+E^{+}}{2}+\frac{E^{-}-E^{+}}{2} \tanh \left[\xi\left(X_{1}+d\right)\right] \\
v\left(X_{1}\right)=\frac{v^{-}+v^{+}}{2}+\frac{v^{-}-v^{+}}{2} \tanh \left[\widehat{\delta}\left(X_{1}+d\right)\right]
\end{gathered}
$$

$$
\begin{gathered}
\beta\left(X_{1}\right)=\frac{\beta^{-}+\beta^{+}}{2}+\frac{\beta^{-}-\beta^{+}}{2} \tanh \left[\widehat{\delta}\left(X_{1}+d\right)\right] \\
k\left(X_{1}\right)=\frac{k^{-}+k^{+}}{2}+\frac{k^{-}-k^{+}}{2} \tanh \left[\widehat{\delta}\left(X_{1}+d\right)\right]
\end{gathered}
$$

where $\left(E^{-}, E^{+}\right)=(1,3),\left(v^{-}, v^{+}\right)=(0.3,0.1),\left(\beta^{-}, \beta^{+}\right)=$ $(0.01,0.03),\left(k^{-}, k^{+}\right)=(1,3), \xi=15, \widehat{\delta}=5$ and $d=0$. Figure 11b shows the temperature distribution over the domain under steady state condition. In order to visualize the gradation in material properties, Eqs. (41)-(44) are plotted across the problem domain as shown in figures 12 and 13. The magnitude of material properties exhibits a sharp jump at the middle of the domain. 


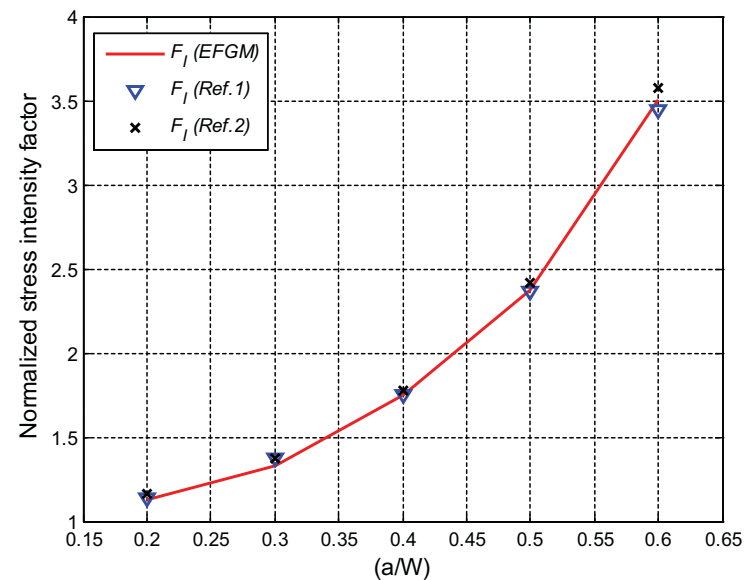

(a)

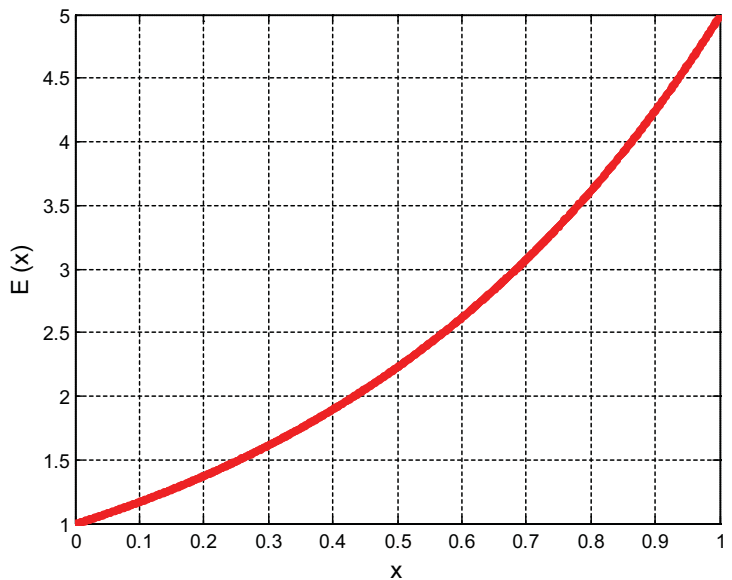

(b)

Figure 7. SIF variation and modulus of elasticity gradation for $E_{2} / E_{1}=5$.

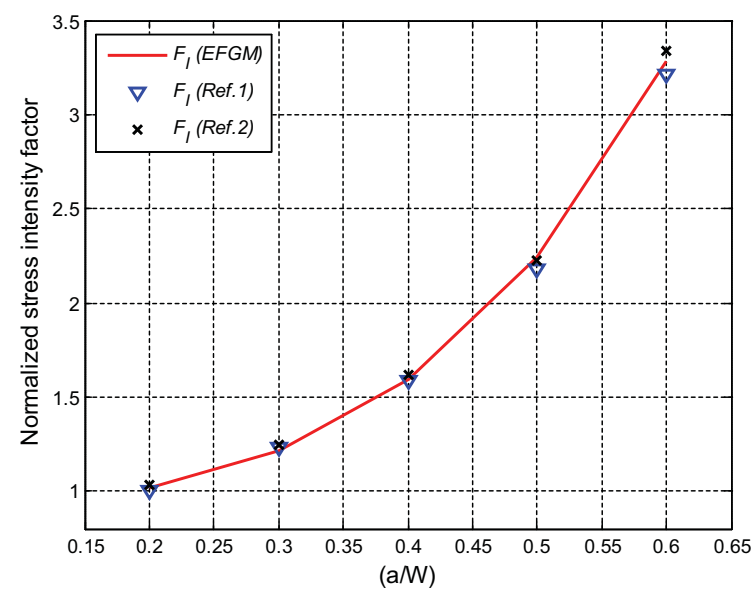

(a)

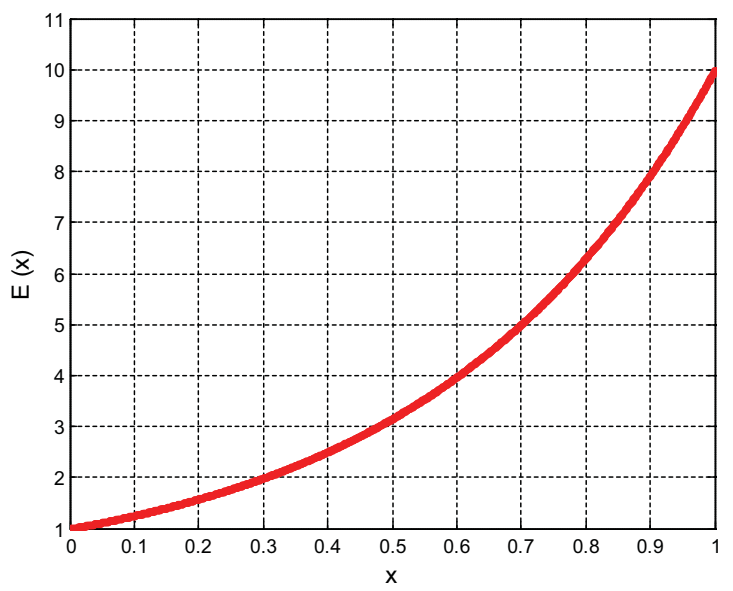

(b)

Figure 8. SIF variation and modulus of elasticity gradation for $E_{2} / E_{1}=10$.

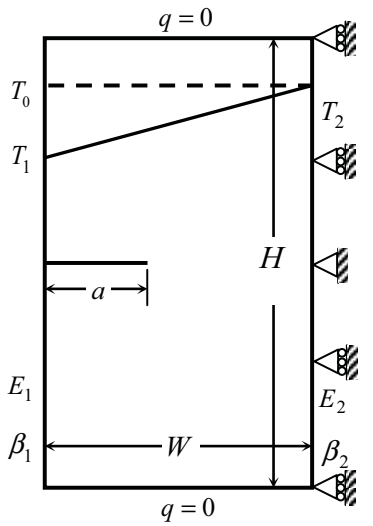

(a)

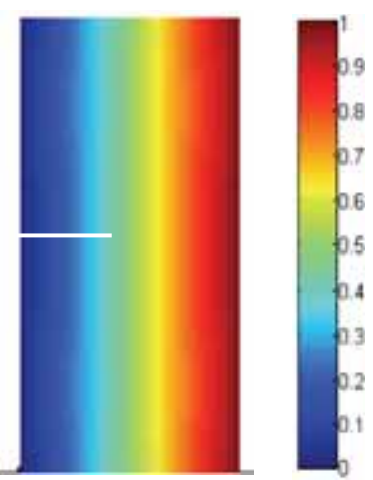

(b)
Figure 9. Problem geometry and temperature distribution over the domain.
Under the prescribed thermal loading and boundary conditions, the edge crack exhibits a mode-I displacement as can be clearly seen from the displaced nodal positions shown in figure $14 \mathrm{a}$. The values of mode-I SIF, i.e. $K_{I}$, have been calculated for different $\left(\frac{a}{W}\right)$ ratios as shown in figure 14b. Again, the EFGM results are found to be in good agreement with the FEM solution [34]. The decrease in value of $K_{I}$ with the increase in crack length is due to the gradation in material properties. The stress fields along $y$ direction $\left(\sigma_{y y}\right)$ plotted in figure $15 \mathrm{a}$ and $\mathrm{b}$ demonstrate the ability of EFG method to capture asymptotic stress fields without any mesh refinement.

7.2c Crack in functionally graded thermal barrier coating $(T B C)$ : Thermal barrier coating (TBC) can be defined as a highly advanced material system usually applied to metallic surfaces operating under elevated temperatures 


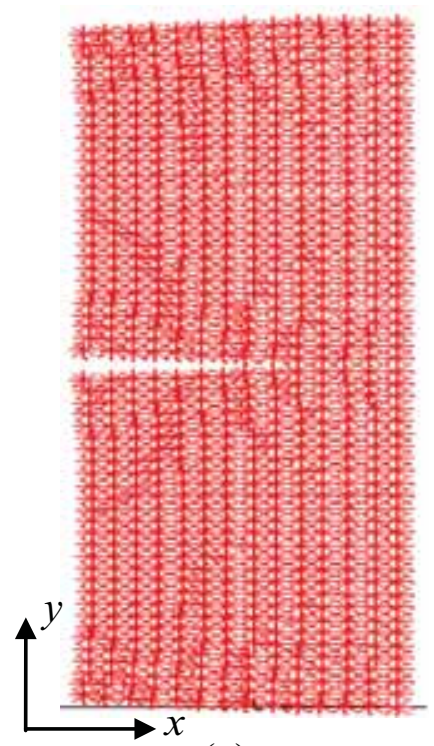

(a)

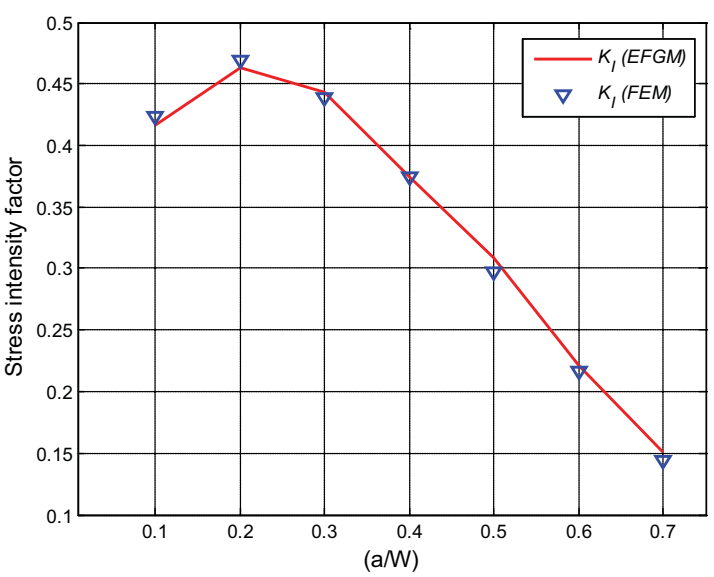

(b)

Figure 10. Displaced nodal positions and variation of stress intensity factor.

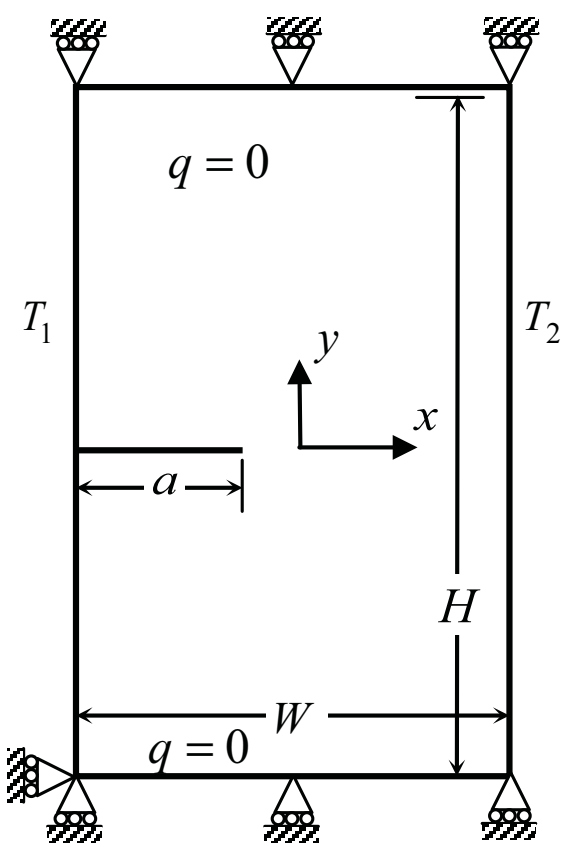

(a)

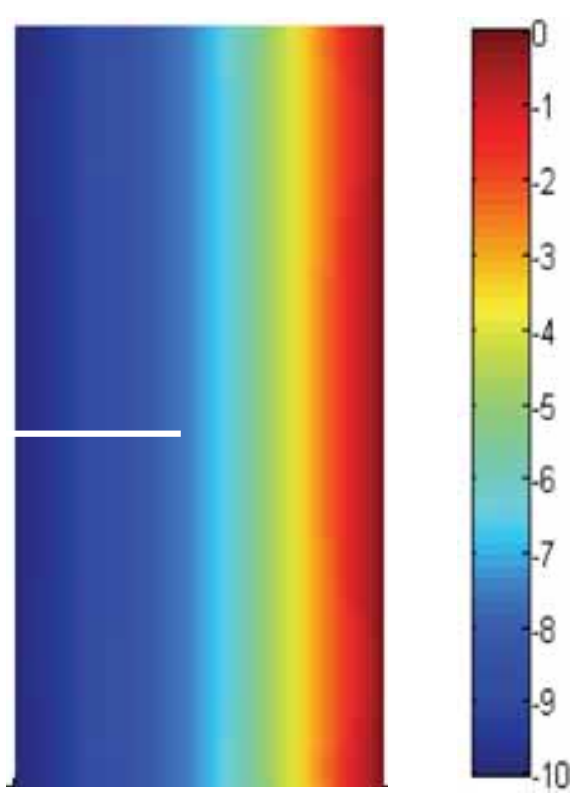

(b)

Figure 11. Problem geometry and temperature distribution over the domain.

such as gas turbine blades, aero-engines, ducts and nozzles, turbocharger casing, and cutting tools. TBCs are characterized by their low thermal conductivity, bearing a large temperature gradient when exposed to heat flow. Modern engineering and industrial applications not only require a restriction on heat transfer but also prevent the surface from the damaging effect of oxidation and corrosion. Since, no single coating composition was able to satisfy all the desired requirements, the idea of coating system came into existence. Functionally graded thermal barrier coating (FTBC) introduces more reliability and reduces interfacial thermal stress between metallic and 


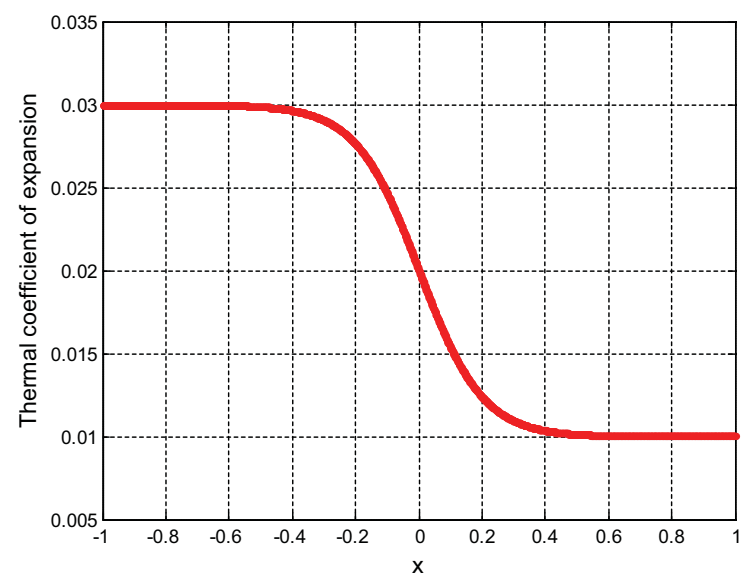

(a)

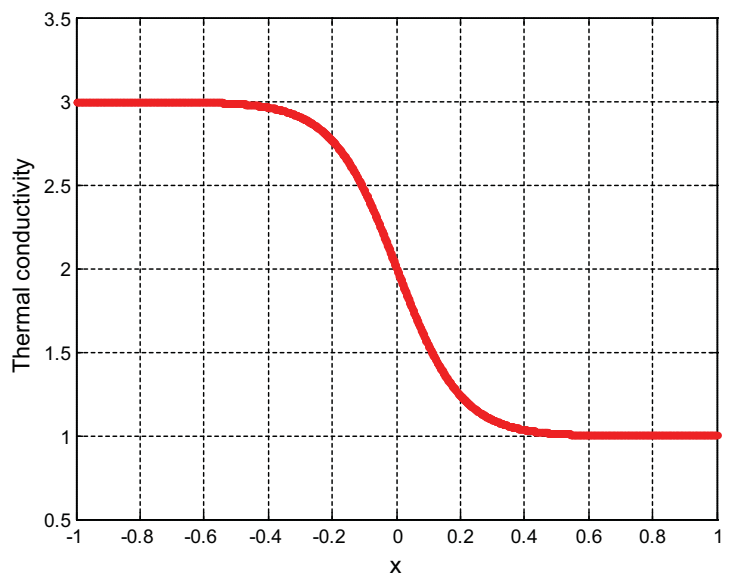

(b)

Figure 12. Gradation of coefficient of thermal expansion and conductivity.

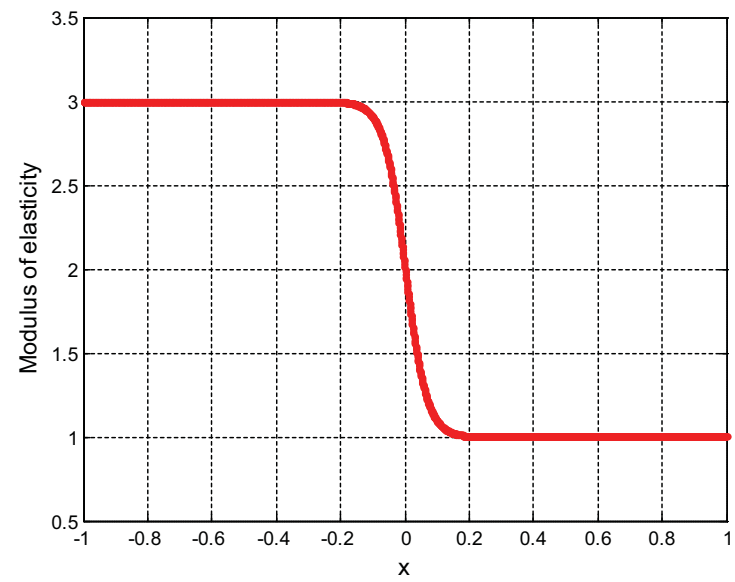

(a)

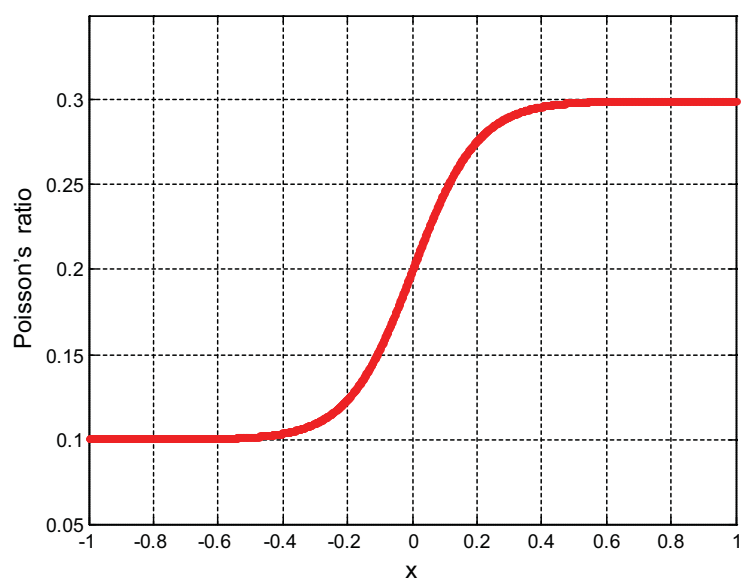

(b)

Figure 13. Gradation of modulus of elasticity and Poisson's ratio.

ceramic layers. FTBC provides less inter-layer thermal stress since the gradient will vary smoothly across the coating thickness. Discontinuities in thermal expansion coefficients between the bond coat and substrate also get reduced. Each FTBC layer will act as a TBC layer with various material compositions; thereby, it gives more life cycles than that of TBC layers of the same thickness under the same loading.

Applications of FTBC at elevated temperature along with corrosive environment make them highly susceptible for crack initiation. In the present problem, an edge crack in FTBC under thermal loading has been modelled and analysed using EFGM. Figure 16 shows a FTBC deposited on the bond coat and the metallic substrate. The FGM coating consists of $100 \%$ zirconium-yttria at $X_{1}=0$ and $100 \%$ nickel-chromium-aluminium-zirconium (NiCrAlY) bond coat at $X_{1}=W_{1}$. The metallic substrate is made up of a nickel-based super-alloy. The material properties of different constituents of TBC are listed in table 1. The dimensions of FGM TBC along with thermal loading and boundary conditions are shown in figure 16. Initially the system is assumed to be at a uniform temperature $\left(T_{0}=1000^{\circ} \mathrm{C}\right)$.

The top and bottom edges of the TBC system are assumed to be insulated. Application of temperature boundary conditions drives the system to a steady state condition with temperature $T_{1}=0.2 T_{0}$ and $T_{2}=0.5 T_{0}$ at left and right edges, respectively. The gradations of 

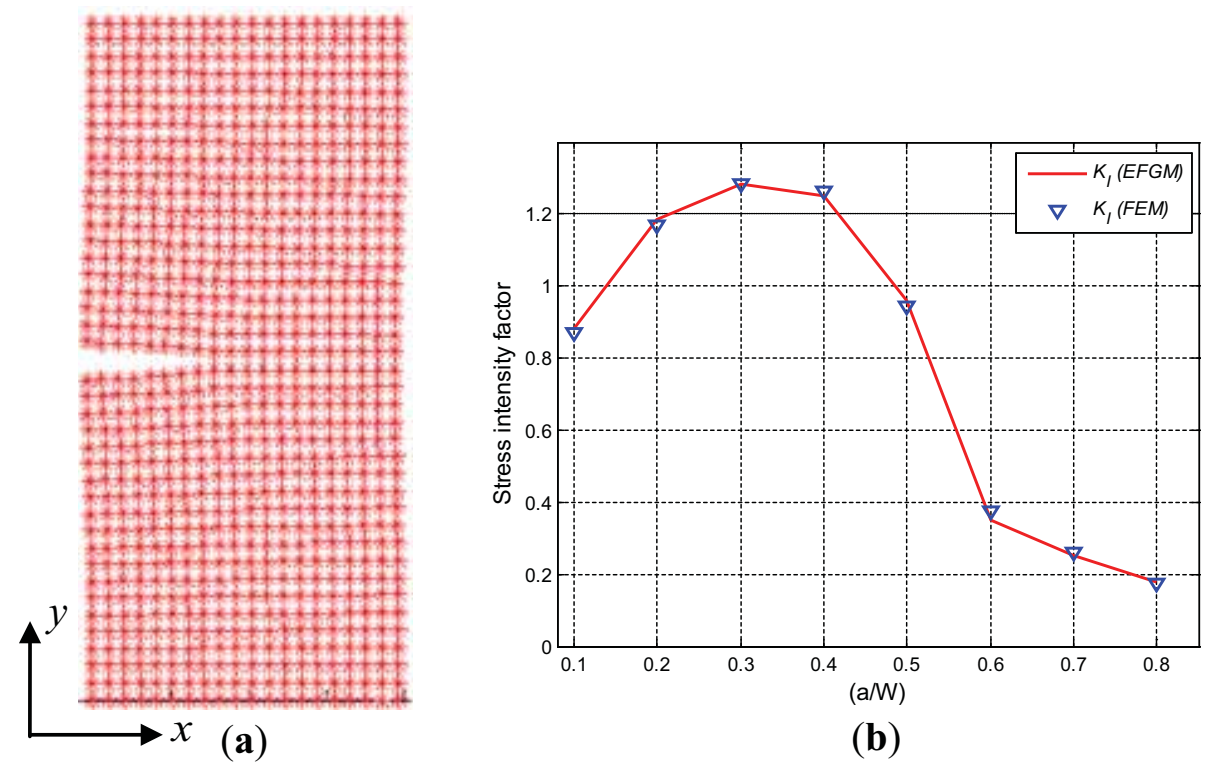

(b)

Figure 14. Displaced nodal positions and variation of stress intensity factor.

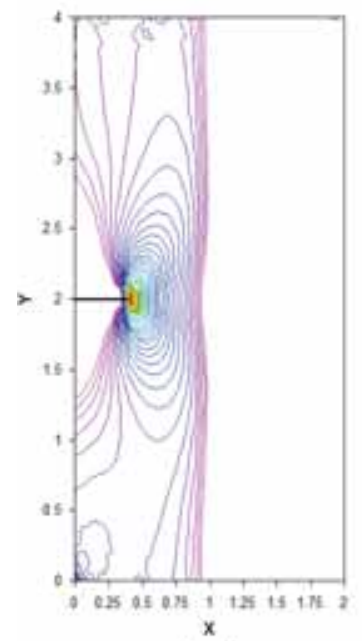

(a)

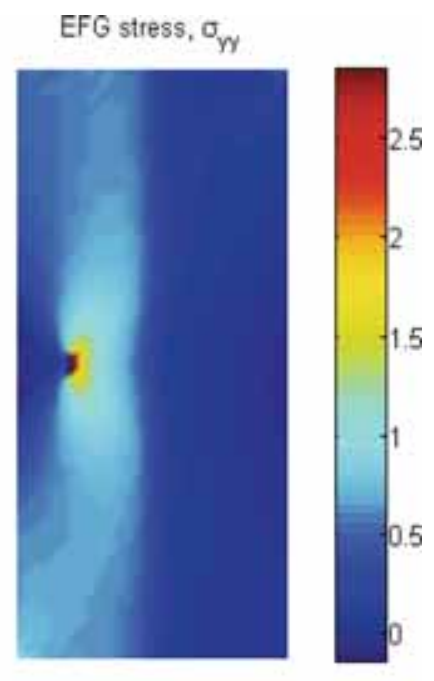

(b)
Figure 15. Stress contours along $y$-direction $\left(\sigma_{y y}\right)$ over the cracked FGM domain with hyperbolic-tangent gradation of material properties.

Young's modulus $(E)$, Poisson's ratio $(v)$ and thermal expansion coefficient $(\beta)$ for the FGM coating region $(0<X<1)$ are defined by the relations given below:

$$
\begin{gathered}
E\left(X_{1}\right)=E_{c}+\left(E_{b c}-E_{c}\right) X^{2} \\
v\left(X_{1}\right)=v_{c}+\left(v_{b c}-v_{c}\right) X \\
\beta\left(X_{1}\right)=\beta_{c}+\left(\beta_{b c}-\beta_{c}\right) X
\end{gathered}
$$

$$
k\left(X_{1}\right)=k_{c}+\left(k_{b c}-k_{c}\right) X^{2}
$$

where subscript $(c)$ symbolizes FGM coating and subscript (bc) denotes the bond coat.

The gradation of material properties along the width of TBC has been shown in figures 17 and 18. Along the width of FGM coating, i.e. $W_{1}$, the modulus of elasticity of the material exhibits a quadratic variation (figure 17a), while Poisson's ratio and coefficient of thermal expansion vary linearly, as shown in figures $17 \mathrm{~b}$ and $18 \mathrm{a}$, respectively. The variation of thermal conductivity is plotted across the width $(W)$ of the TBC as shown in figure 18b. From figure 18b, it can be clearly observed that up to the thickness of FGM coating $\left(W_{1}\right)$, the thermal conductivity follows a quadratic variation, and its value reaches a maximum at the bond coat layer. At the interface of bond coat and metal substrate, there is a sharp decline in the magnitude of the thermal conductivity, and further, its value remains constant for the remaining thickness of metal substrate.

A uniform nodal density of 1250 nodes along with sixpoint Gauss quadrature has been used to simulate the problem under plane strain conditions. Temperature field remains unaffected by the presence of crack as shown in figure 19 as the heat flux is parallel to the crack surface. Figure 20a shows the variation of normalized temperature, i.e. $\left(\frac{T\left(X_{1}\right)}{T_{0}}\right)$, along the width of TBC assembly. A quadratic variation of thermal conductivity along the width of FGM layer is quite justified by a resulting quadratic variation of temperature as can be clearly seen from figure $20 \mathrm{a}$. The values of mode-I SIF, i.e. $K_{I}$, are calculated for different 


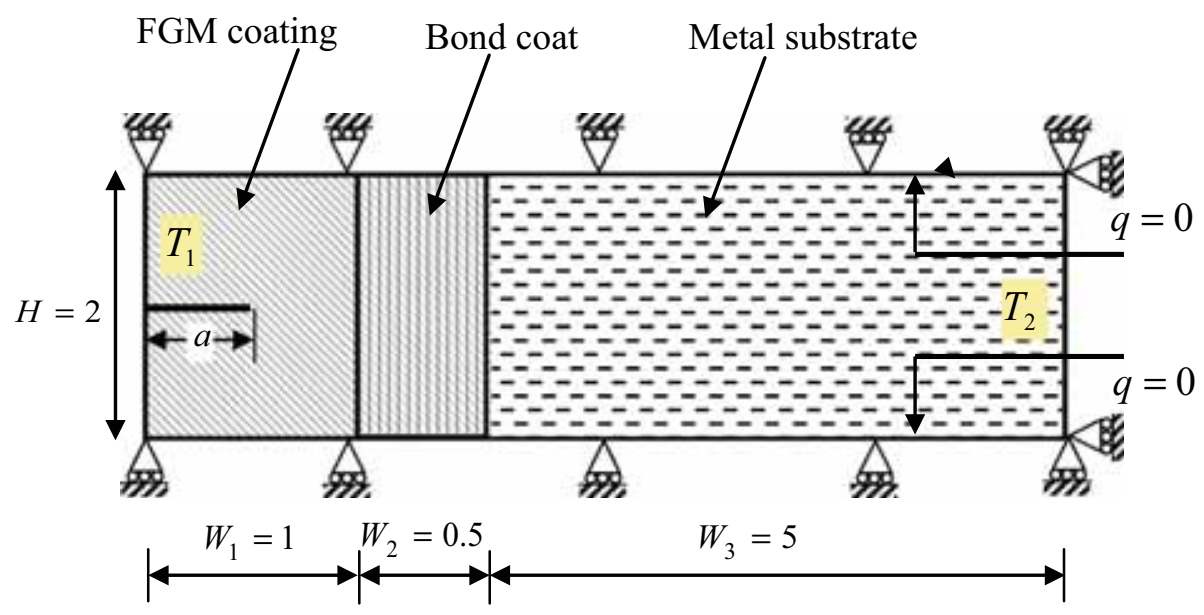

Figure 16. A crack in a functionally graded thermal barrier coating.

Table 1. Properties of TBC constituents.

\begin{tabular}{lccc}
\hline Material property & Zirconium-yttria (FGM) & Bond coat NiCrAlY & Metallic substrate (Ni) \\
\hline$E(\mathrm{GPa})$ & 27.6 & 137.9 & 175.8 \\
$v$ & 0.25 & 0.27 & 0.25 \\
$\beta\left({ }^{\circ} \mathrm{C}^{-1}\right)$ & $10.01 \times 10^{-6}$ & $15.16 \times 10^{-6}$ & $13.91 \times 10^{-6}$ \\
$k(\mathrm{~W} / \mathrm{mK})$ & 1 & 25 & 7 \\
\hline
\end{tabular}

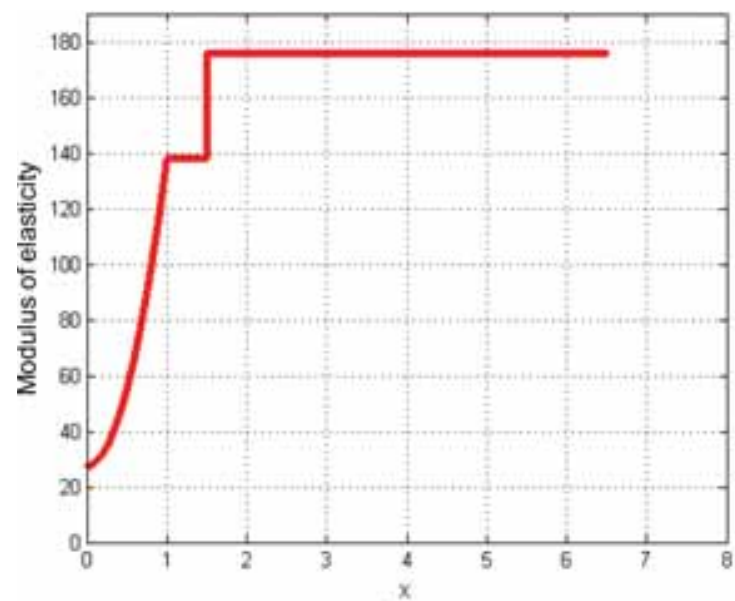

(a)

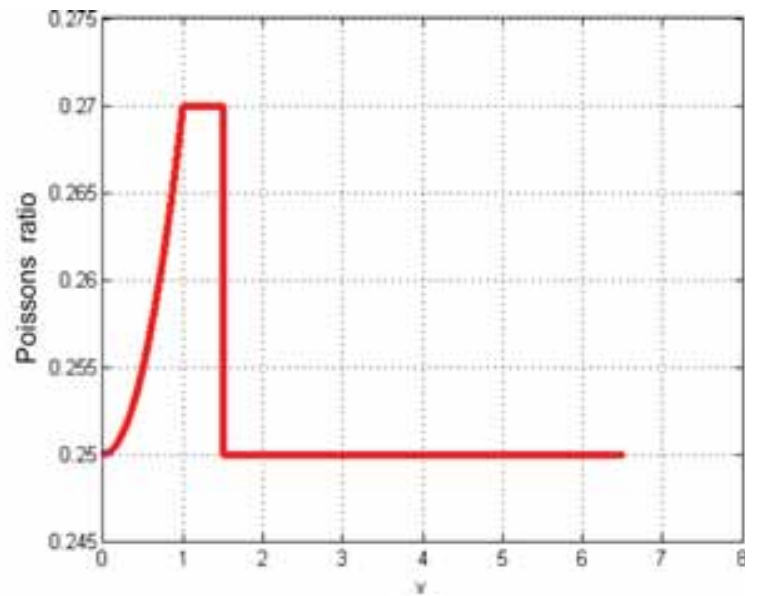

(b)

Figure 17. Gradation of modulus of elasticity and Poisson's ratio across the width $(W)$ of the domain.

$\left(\frac{a}{W}\right)$ ratios as shown in figure 20b. A good agreement in numerical results with the FEM solution [34] demonstrates the modelling capability of EFGM. In order to visualize the presence of crack, the stress-strain field contours have been generated for the FTBC assembly for a ratio of $a / W=0.4$ as shown in figures 21 and 22.

\section{Conclusion}

In the present work, EFGM has been implemented for modelling thermal fracture in FGMs. Two different cases of property gradation were considered for an edge crack problem along with simulating crack in a thermal barrier 


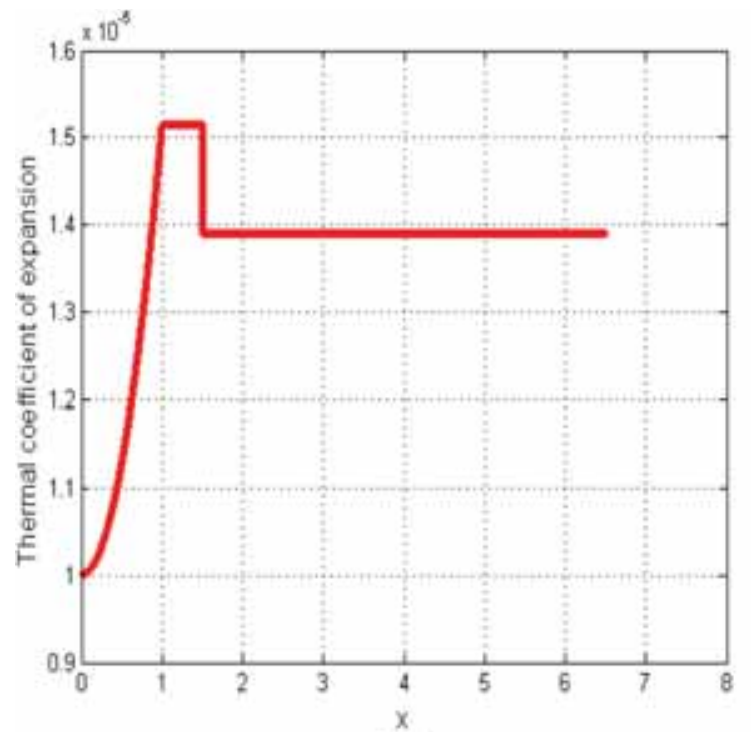

(a)

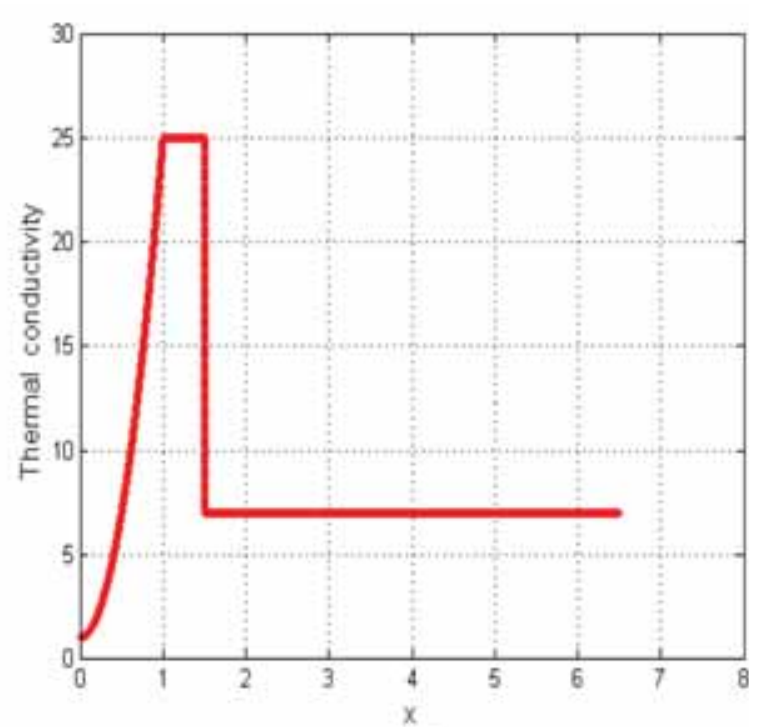

(b)

Figure 18. Gradation of coefficient of thermal expansion and conductivity across the width $(W)$ of the domain.
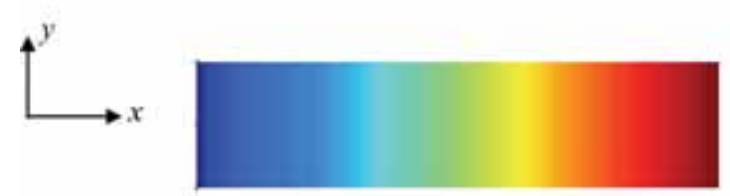

Figure 19. Temperature distribution over the cracked $\mathrm{TBC}$ assembly. coating (TBC). The values of stress intensity factors (SIFs) were calculated using a modified form of interaction integral that accounts for both gradation of material properties and the applied temperature field. A comparison of SIF values predicted by EFGM and available reference solutions generated analytically or numerically reveals the applicability of present work. The present simulation and analysis establishes EFGM as an efficient method for modelling cracks in FGMs and reflects its potential to solve a variety of practical fracture problems. The simplicity and accuracy of this implementation show that it can be further

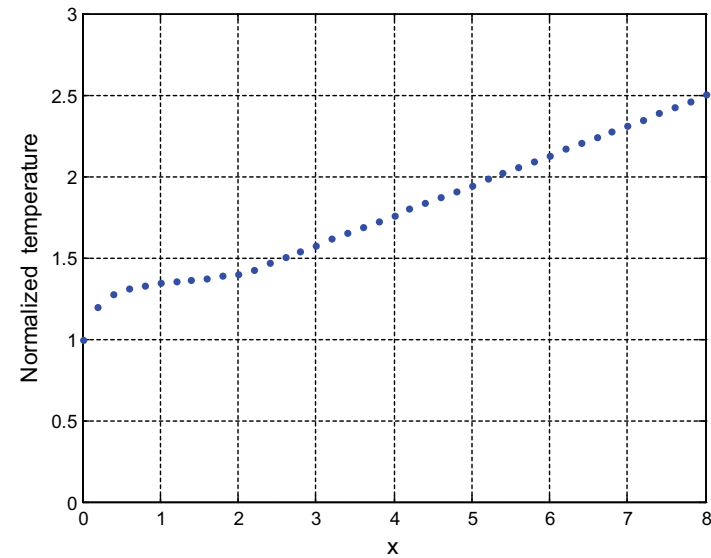

(a)

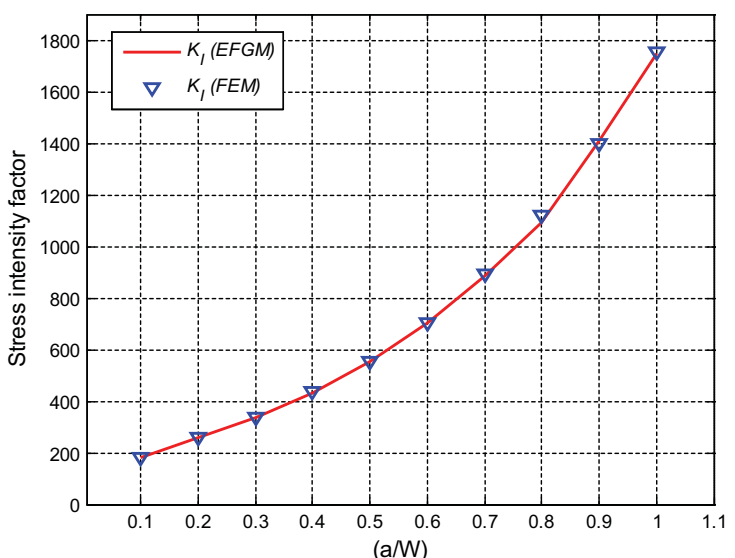

(b)

Figure 20. Variation of normalized temperature and stress intensity factor. 


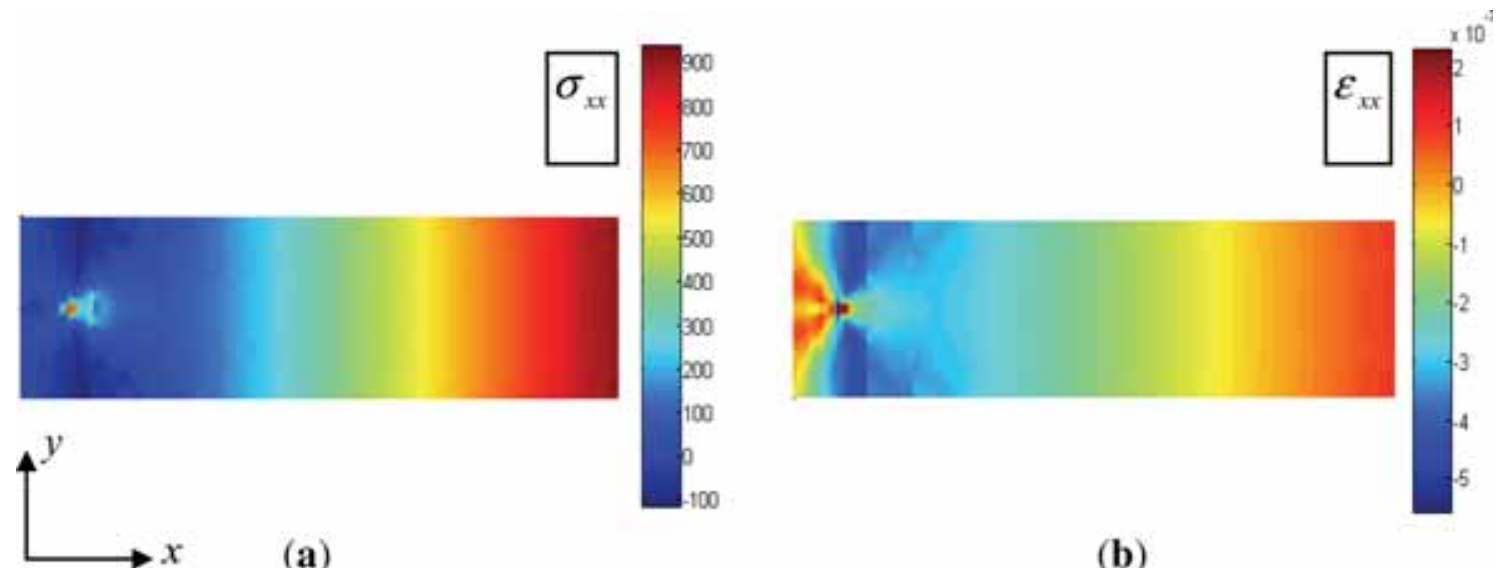

Figure 21. Stress-strain contours along $x$-direction over the cracked TBC domain.

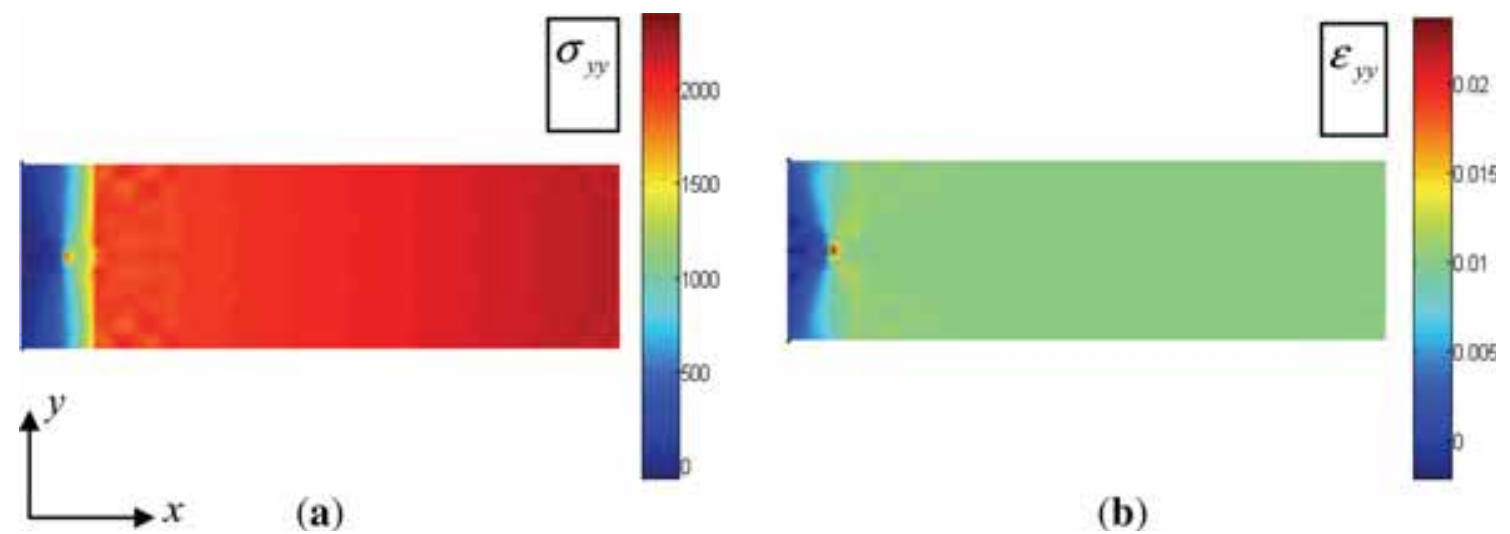

Figure 22. Stress-strain contours along $y$-direction over the cracked TBC domain.

extended to study and analyse the fracture in graded materials with multi-loading and complex geometry conditions.

\section{References}

[1] Koizumi M 1997 FGM activities in Japan. Compos. Part B: Eng. 8368: 1-4

[2] Barthel K and Rambert S 1999 Thermal spraying and performance of graded composite cathodes as SOFC-component. Mater. Sci. Forum 308: 800-805

[3] Hart N T, Brandon N P, Day M J and Shemilt J E 2001 Functionally graded cathodes for solid oxide fuel cells. $J$. Mater. Sci. 36(5): 1077-1085

[4] Liu Y, Compson C and Liu M 2004 Nanostructured and functionally graded cathodes for intermediate temperature solid oxide fuel cells. J. Power Sources 138(1): 194-198

[5] Coomar N and Kadoli R 2010 Comparative analysis of steady state heat transfer in a TBC. Sadhana-Acad. Proc. Eng. Sci. 35(1): 1-17
[6] Gibson R E 1967 Some results concerning displacements and stresses in a non-homogeneous elastic half-space. Geotechnique 17(1): 58-67

[7] Atkinson C and List R D 1978 Steady state crack propagation into media with spatially varying elastic properties. Int. J. Eng. Sci. 16(10): 717-730

[8] Dhaliwal R S and Singh B M 1978 On the theory of elssticity of a nonhomogeneous medium. J. Elast. 8(2): 211-219

[9] Delale F and Erdogan F 1983 The crack problem for a nonhomogeneous plane. J. Appl. Mech. 50(3): 609-614

[10] Jin Z H and Noda N 1993 An internal crack parallel to the boundary of a nonhomogeneous half plane under thermal loading. Int. J. Eng. Sci. 31(5): 793-806

[11] Erdogan F 1995 Fracture mechanics of functionally graded materials. Compos. Eng. 5(7): 753-770

[12] Gu P and Asaro R J 1997 Cracks in functionally graded materials. Int. J. Solids Struct. 34(1): 1-17

[13] Gu P, Dao M and Asaro R J 1999 A simplified method for calculating the crack-tip field of functionally graded materials using the domain integral. J. Appl. Mech. 66(1): 101-108 
[14] Chen J, Wu L and Du S 2000 A modified J integral for functionally graded materials. Mech. Res. Commun. 27(3): 301-306

[15] Marur P R and Tippur H V 2000 Numerical analysis of crack-tip fields in functionally graded materials with a crack normal to the elastic gradient. Int. J. Solids Struct. 37(38): 5353-5370

[16] Kim J and Paulino G H 2002 Finite element evaluation of mixed mode stress intensity factors in functionally graded materials. Int. J. Numer. Methods Eng. 53(8): 1903-1935

[17] Chi S and Chung Y 2003 Cracking in coating-substrate composites with multi-layered and FGM coatings. Eng. Fract. Mech. 70: 1227-1243

[18] Rangaraj S and Kokini K 2003 Estimating the fracture resistance of functionally graded thermal barrier coatings from thermal shock tests. Surf. Coat. Technol. 173(3): 201-212

[19] Çalliŏglu H 2011 Stress analysis in a functionally graded disc under mechanical loads and a steady state temperature. Sadhana - Acad. Proc. Eng. Sci. 36(February): 53-64

[20] Saucedo-mora L, Sláme K, Thandavamoorthy U and Marrow T J 2015 Multi-scale modeling of damage development in a thermal barrier coating. Surf. Coat. Technol. 276: 399-407

[21] Afsar A M and Song J I 2010 Effect of FGM coating thickness on apparent fracture toughness of a thick-walled cylinder. Eng. Fract. Mech. 77(14): 2919-2926

[22] Rao B N and Rahman S 2003 Mesh-free analysis of cracks in isotropic functionally graded materials. Eng. Fract. Mech. 70(1): $1-27$

[23] Dai K Y, Liu G R, Han X and Lim K M 2005 Thermomechanical analysis of functionally graded material (FGM) plates using element-free Galerkin method. Comput. Struct. 83(17-18): 1487-1502

[24] Dolbow J E and Gosz M 2002 On the computation of mixedmode stress intensity factors in functionally graded materials. Int. J. Solids Struct. 39(9): 2557-2574

[25] Natarajan S, Baiz P M, Bordas S, Rabczuk T and Kerfriden P 2011 Natural frequencies of cracked functionally graded material plates by the extended finite element method. Compos. Struct. 93(11): 3082-3092

[26] Bayesteh H and Mohammadi S 2013 XFEM fracture analysis of orthotropic functionally graded materials. Compos. Part B Eng. 44(1): 8-25

[27] Belytschko T, Lu Y Y and Gu L 1994 Element free Galerkin methods. Int. J. Numer. Methods Eng. 37(2): 229-256

[28] Belytschko T and Fleming M 1999 Smoothing, enrichment and contact in the element-free Galerkin method. Comput. Struct. 71(2): 173-195

[29] Belytschko T, Krongauz Y, Fleming M, Organ D and Snm Liu W K 1996 Smoothing and accelerated computations in the element free Galerkin method. J. Comput. Appl. Math. 74(1-2): 111-126

[30] Sladek J and Sladek V 1997 Evaluation of T-stresses and stress intensity factors in stationary thermoelasticity by the coservation integral method. Int. J. Fract. 86(3): 199-219

[31] Paulino G H and Kim J H 2005 Consistent formulations of the interaction integral method for fracture of functionally graded materials. J. Appl. Mech. 72(3): 351-364

[32] Wilson W K and Yu I W 1979 The use of the J-integral in thermal stress crack problems. Int. J. Fract. 15(4): 377-387

[33] Rice J R 1968 A path independent integral and the approximate analysis of strain concentration by notches and cracks. J. Appl. Mech. 35(2): 379

[34] Amit K C and Kim J H 2008 Interaction integrals for thermal fracture of functionally graded materials. Eng. Fract. Mech. 75(8): 2542-2565

[35] Erdogan F and Wu B H 1997 The surface crack problem for a plate with functionally graded properties. J. Appl. Mech. 64(3): 449-456

[36] Chen J, Wu L Z and Du S Y 2000 Element-free Galerkin methods for fracture of functionally-graded materials. Key Eng. Mater. 183: 487-492

[37] Duflot M 2008 The extended finite element method in thermoelastic fracture mechanics. Int. J. Numer. Methods Eng. 74(5): 827-847 